\title{
Isolation and identification of Mycoplasma capricolum subsp. capripneumoniae from goats
}

\author{
Iqra Naseem ${ }^{1 *}$, Quratulain Amin ${ }^{1}$, Syed Faizan Haider Gardezi ${ }^{2}$, Muhammad Fakhar-i-Adil ${ }^{3}$, Sajjad ur Rehman ${ }^{1}$
}

${ }^{1}$ Institute of Microbiology, University of Agriculture, Faisalabad, Pakistan.

${ }^{2}$ Department of Pathology, University of Agriculture, Faisalabad, Pakistan.

${ }^{3}$ Department of Anatomy, University of Agriculture Faisalabad, Pakistan.

*Correspondence Author's email: iqra.live9@ gmail.com

\begin{abstract}
Contagious caprine pleuropneumonia is a transmissible disease in goats caused by Mycoplasma capricolum subsp. capripneumoniae (Mccp). The disease is responsible for remarkable morbidity and mortality in goats. In Pakistan, data regarding its prevalence and incidence is lacking. This research study was aimed at providing the current prevalence rate in Punjab, Pakistan. For this, 50 samples including pleural fluids, nasal swabs and trachea were collected from slaughterhouses, livestock markets and veterinary hospitals around Faisalabad and processed for the determination of Contagious caprine pleuropneumonia by culture and confirmation by polymerase chain reaction. Characteristics fried egg colonies and Giemsa staining were indicative of Mccp. The culture positive samples were then processed to detect 16S rRNA gene of Mccp. Based on cultural and molecular evidence: a) The prevalence of Contagious caprine pleuropneumonia was $40 \%$ in slaughterhouse specimens, b) The prevalence of contagious caprine pleuropneumonia was $46.66 \%$ in livestock markets and, c) The prevalence of contagious caprine pleuropneumonia was $15 \%$ in veterinary hospitals.
\end{abstract}

Keywords: Mccp, Goat, Polymerase chain reaction

\section{Highlights}

$>\quad$ The study was aimed at providing a figure for prevalence of contagious caprine pleuropneumonia in the goat population in Faisalabad Pakistan.

$>\quad$ The detection of the disease organism was confirmed by bacterial culturing and biochemical testing.

$>\quad$ The culture positive samples were further confirmed via qualitative polymerase chain reaction targeting the $16 \mathrm{~S}$ rRNA gene.

$>$ The prevalence of contagious caprine pleuropneumonia was found to be $40 \%, 46.66 \%$, and $15 \%$ in slaughterhouse specimens, livestock markets, and veterinary hospitals.

$>\quad$ The prevalence was found to be highest in samples collected from younger animals as compared to adult ones.

\section{INTRODUCTION}

Contagious caprine pleuropneumonia, caused by Mycoplasma capricolum subsp. capripneumoniae (Mccp), is a fatal disease of goats that causes a significant economic loss in the Middle East, Africa, Eastern Europe and Asia (AbdElrahman, Khafaga, \& Abas, 2020). Mccp belongs to the cluster of Mycoplasma mycoides which consists of six mycoplasma species including M. capricolum, M. capripneumoniae, M. mycoides SC, M. mycoides LC, Mycoplasma sp. Bovine serogroup 7 and M. capri (Shah, et al., 2017, Noah, et al., 2011). M. capripneumoniae affect goats only (OIE, 2008). Lesions are confined to the thoracic cavity with straw-colored fluid in the pleural cavity at necropsy and lesions are more specific in lungs and pleura. In severe cases, lungs are consolidated and hepatized with the port wine color (Abraham, et al., 2015). In chronic cases, lung tissues are black and show necrotization (Rahman, et al., 2018). Classical contagious caprine pleuropneumonia lesions are confined to the thoracic cavity while the lesions caused by Mycoplasma mycoides LC, M. capri, and M. capricolum can be in any other parts of the body including the thoracic cavity (El-Deeb, et al., 2017).

Confirmatory diagnosis of $M$. capripneumoniae (Mccp) is very difficult because it has cross-reactivity with other mycoplasma cluster's species (Namazi, Derakhshandeh, Hezaveh, \& Eraghi, 2020). Although isolation and identification steps are important in confirmation of Mycoplasma capricolum subsp. capripneumoniae it also requires advanced laboratory facilities as well as experience to treat this luxuriant bacterium (Kabir and Bari, 2015). Similarly, the use of antibiotics also becomes a hindrance in the isolation of M. capripneumoniae. Molecular technique especially PCR is useful in the confirmatory diagnosis of M. capripneumoniae (Woubit, et al., 2011). An accurate molecular technique allows to identify Mccp by making multiple copies of $16 \mathrm{~S}$ gene.

The aim of this study was to provide a baseline prevalence data of Mccp in Faisalabad based on molecular characterization. 


\section{MATERIALS AND METHODS}

\section{1. $\quad$ Sample Collection and Processing}

Samples were collected aseptically from 50 goats showing respiratory signs and nasal discharge as shown both age-wise and sex-wise in Table 1 and 2, respectively.

Table 1: Age wise sampling of animals from different collection centers

\begin{tabular}{cccc}
\hline Age & $\begin{array}{c}\text { Abattoirs } \\
\text { (Lung's exudate) }\end{array}$ & $\begin{array}{c}\text { Livestock markets } \\
\text { (Nasal swabs) }\end{array}$ & $\begin{array}{c}\text { Veterinary hospitals } \\
\text { (Nasal swabs) }\end{array}$ \\
\hline 2-6 months & 8 & 9 & 10 \\
7-12 months & 4 & 3 & 6 \\
Above 12months & 3 & 3 & 4 \\
Total & 15 & 15 & 20 \\
\hline
\end{tabular}

Table 2: Sex wise distribution of the collected samples

\begin{tabular}{ccc}
\hline Source & Male & Female \\
\hline Abattoirs & 8 & 7 \\
Livestock markets & 7 & 8 \\
Veterinary hospitals & 12 & 8 \\
\hline
\end{tabular}

Immediately after collection, sample swabs were inoculated in Hay's flick broth poured in McCartney bottles and incubated at $37^{\circ} \mathrm{C}$ for $24-48$ hours following the procedure of Kabir and Bari, 2015. A second passage was given to positive samples in Hay's flick broth in McCartney bottles at $37^{\circ} \mathrm{C}$ for 24 hours (Ahmad et al., 2020).

\subsection{Purification of organism}

After second passage, the material was further streaked on Hay's flick agar and placed in anaerobic jar at $37^{\circ} \mathrm{C}$ for $2-3$ days. The colony characters were recorded as there were off-white color growth on agar plates. Colonies were identified under microscope at 100X. Purified colonies were proceeded for Geimsa staining and biochemical characterization via glucose fermentation, phosphatase activity and Tetrazolium reduction tests as described (Parray et al., 2019).

\subsection{Molecular characterization}

The colonies on the Hay's flick agar media were processed to extract DNA of the organism using GF-1 nucleic acid extraction kit according to manufacturer'sinstructions. Species specific forward and reverse primer against 16S rRNA gene of Mccp, (MmF 5'-CGAAAGCGGCTTACTGGCTTGTT-3') and (MmR 5'-TTGAGATTAGCTCCCCCTCACAG-3') respectively, were used for the confirmation of Mycoplasma capricolum subsp. capripneumoniae via qualitative polymerase chain reaction. 35 cycles of PCR were carried out under the following conditions mentioned in table 3.

Table 3: Conditions for polymerase chain reaction

\begin{tabular}{ccc}
\hline Step Name & Temperature & Time \\
\hline Initial Denaturation & $94^{\circ} \mathrm{C}$ & 4 minutes \\
Denaturation & $95^{\circ} \mathrm{C}$ & 45 seconds \\
Annealing & $55^{\circ} \mathrm{C}$ & 1 minute \\
Elongation & $72^{\circ} \mathrm{C}$ & 1 minute \\
Final elongation & $72^{\circ} \mathrm{C}$ & 7 minute \\
\hline
\end{tabular}

\section{RESULTS}

A total of 50 samples were collected from abattoirs, livestock market, and veterinary hospitals. The samples were processed for culture and molecular characterization of Mccp. Of the 50 samples processed, 16 samples gave positive result following 2 passages in Hay's flick broth, the positive specimens were indicated by red color (Fig 1).

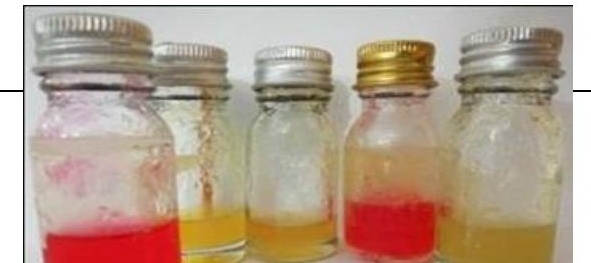


Fig. 1: Hay's Flick broth showing positive (yellow) and negative (red) samples in McCartney bottles.

Broth positive samples were inoculated then on Hay's flick agar. Off white color growth obtained on gar media was indicative of Mccp (Fig 2).

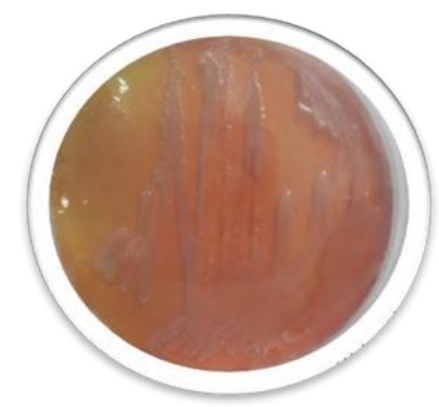

Fig. 2: Off-white colonies on Hay's flick agar.

The colonies were stained with giemsa's stain and analyzed under microscope at 100X magnification to observe fried egg like colonies as shown in figure 3.

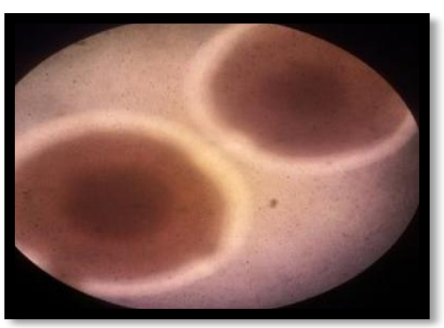

Fig 3. Microscopic observation - Fried egg appearance of colonies.

\section{1. $\quad$ Source-based isolation of Mccp}

Of the abattoir'ssamples, 40 percent were culture positive for Mccp compared to 46.66 percent culture positive in samples from livestock markets and 15 percent culture positive in samples from Veterinary Hospitals (Table 4). The difference in prevalence of the disease between sources was non-significant (Chi square value 4.861 , df 2 , $\mathrm{P}$ value $=0.088$ ).

Table 4: Mccp culture positive cases in Goats in abattoirs, livestock markets and Veterinary Hospitals.

\begin{tabular}{cccc}
\hline Source of Samples & Number of samples & $\begin{array}{c}\text { Number of positive } \\
\text { samples }\end{array}$ & $\begin{array}{c}\text { Percentage of positive } \\
\text { samples }\end{array}$ \\
\hline Abattoirs & 15 & 6 & 40 \\
Livestock markets & 15 & 7 & 46.66 \\
Veterinary hospitals & 20 & 3 & 15 \\
Total samples & 50 & 16 & 32 \\
\hline
\end{tabular}

\subsection{Age-based isolation of Mccp}

The prevalence was 39.19 percent in abattoirs relative to 29.41 percent in livestock markets and 20 percent in veterinary hospitals. There was non- significant difference in disease prevalence between different age groups (Table 5) (Chi square 1.293 , df 2 , $\mathrm{P}$ value $=0.524)$. 
Table 5: Age wise distribution of Mccp culture positive in suspect goats.

\begin{tabular}{cccc}
\hline Age (months) & $\begin{array}{c}\text { Number of } \\
\text { samples }\end{array}$ & $\begin{array}{c}\text { Number of } \\
\text { positive samples }\end{array}$ & $\begin{array}{c}\text { Percentage of } \\
\text { positive samples }\end{array}$ \\
\hline 2 to 6 months & 23 & 9 & 39.13 \\
6 to 12 months & 17 & 5 & 29.41 \\
Above 12 months & 10 & 2 & 20 \\
Total & 50 & 16 & - \\
\hline
\end{tabular}

\subsection{Sex-based isolation of Mccp}

The prevalence of CCPP was 25.92 percent in male and 39.13 percent in female goats. Prevalence of disease did not significantly differ between male and female (Table 6). (Chi square $=0.995$, df $1, \mathrm{P}$-value $=0.319$ )

Table 6: Sex-based distribution of Mccp culture positive cases in suspect goats.

\begin{tabular}{llll}
\hline Sex & $\begin{array}{l}\text { Number of } \\
\text { samples }\end{array}$ & $\begin{array}{l}\text { Number of } \\
\text { positive samples }\end{array}$ & $\begin{array}{l}\text { Percentage of } \\
\text { positive samples }\end{array}$ \\
\hline Male & 27 & 7 & 25.92 \\
Female & 23 & 9 & 39.13 \\
Total & 50 & 16 & - \\
\hline
\end{tabular}

\subsection{Molecular Detection of Mccp through PCR}

Of the 50 samples, 16 were culture positive for Mycoplasma capricolum subsp. capripneumoniae. All of these were positive for 16S rRNA gene (fig.4) confirming Mycoplasma capricolum subsp. capripneumoniae. Product size for this PCR was 548bp that was compared by using $1 \mathrm{~kb}$ DNA ladder.

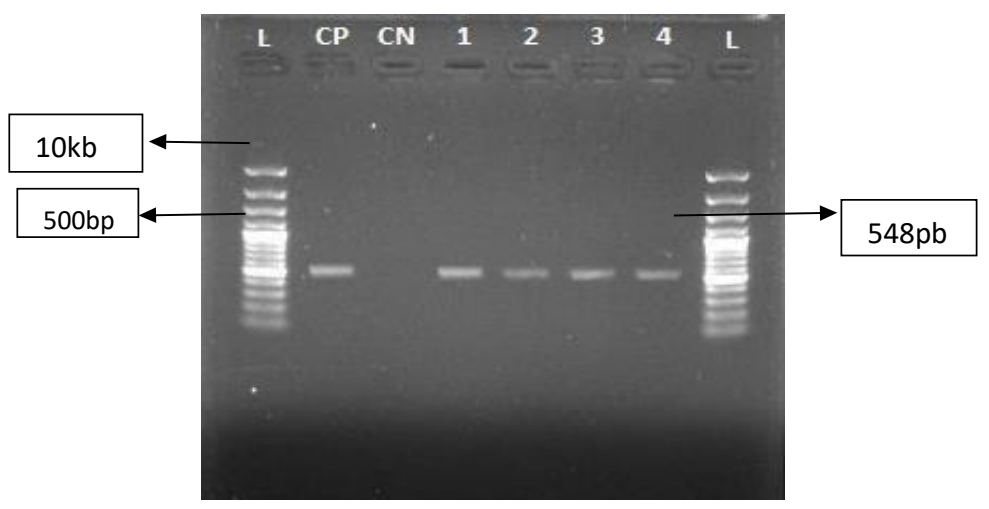

Fig 4. Detection of gene of 16S rRNA of Mycoplasma via Qualitative PCR

\section{DISCUSSION}

Serologically using the cELISA kit, CCPP has been reported in many countries around the world including Ethiopia 14.6 per cent, Tajikstan 10.1 per cent, Pakistan Diameer 44.2 per cent and Gilgit 2.7 per cent; Mauritius 16 per cent and Kenya 6-90 per cent (ContPeyraud et al., 2014). CCPP is a common disease in goats widely prevalent in Pakistan. In the past, some reports from Punjab (Shahzad et al., 2016), Baluchistan (Awan et al., 2009, Ejaz et al., 2015) and mostly from Khyber Pakhtoon Khaw (KPK) reported cELISA-based seroprevalence. This study has concentrated on Mycoplasma capricolum subsp. capripneumoniae (Mccp) isolation from goats clinically showing fever with respiratory signs. This study finds that (Mccp) can be isolated from a high number of goats clinically showing low grade fever and respiratory signs.

Of the collected samples, 32 percent were culture and PCR positive for Mccp (Table-3). Many studies report seroprevalence rather than Mccp isolation, hence present findings cannot be compared to previous studies. In a study during 2006- 2007, Shahzad et al., (2012) used Latex Agglutination test (LAT) to detect the prevalence of CCPP in different regions of Pakistan but found zero prevalence of Mccp. Similarly, Ejaz et al. (2015) designed a study in goats for molecular prevalence of mycoplasmosis in different districts of Baluchistan and found not a single positive case for Mccp. However, Hussain et al. (2012), in line with the present study. reported a 32 per cent sero-prevalence of CCPP in Beetal goats by counter electrophoresis technique. This diversity of observations could be due to non-specificity or non-sensitivity of the 
tests used.

In the present study, Mccp isolation was higher in samples from livestock markets and slaughterhouse compared to samples from Veterinary Hospitals (Table-3).

This could be due to two factors: a) high outlet of non-productive animals suffering from respiratory disease to the livestock markets and slaughterhouse and b) Use of antibiotics could interfere in Mccp isolation in goats brought to the Veterinary Hospitals. No other study is available to compare this finding. (Shahzad et al., 2016), using cELISA, reported 33.33 percent seroprevalence of CCPP.

In the present study, Mccp isolation rate was highest (39.19\%) in 2-6 months age group followed by 29.41 per cent in 6-12 months age group and 20 per cent in above 12 months of age group (Table-4). It appears that the probability of isolating Mccp is greater from younger compared to older animals. This hypothesis is supported by Wazir et al. who reported a higher prevalence of Mccp using cELISA in kids (6.73\%) and young goats (3.85\%) compared to adult goats $(2 \%)$ in Khyber Pakhtoon Khaw (KPK).

In the present study, Mccp was isolated from 39.13 percent in females compared with 25.92 percent in males (Table5). This is comparable to Shahzad et al. (2012) and (Teshome, Sori, Sacchini, \& Wieland, 2019) reported a higher prevalence in females compared to males in different areas of Pakistan (Thakur, Sharma, Verma, \& Kanwar, 2019) reported 16.1 percent prevalence in female compared to 10.7 percent in male.

In Summary, the study suggests that CCPP is widely distributed in goats around Faisalabad. Samples from young animals i.e., less than 6 months of age, may provide higher culture positive samples.

\section{Acknowledgements}

I am grateful to my supervisor Prof. Dr. Sajjad-ur-Rehman who provided me with all the facilities and platform to conduct the research. I pay my best regards to Prof. Dr. Ahmed Din Anjum, who helped me in the technical write up. My gratitude is also extended to Prof. Dr. Saqib, who helped me in providing consumables, and some other materials for the conduction of the research.

\section{Conflict of interest}

The authors have no conflict of interest to disclose.

\section{References}

Abd-Elrahman, A. H., Khafaga, A. F., \& Abas, O. M. (2020). The first identification of contagious caprine pleuropneumonia (CCPP) in sheep and goats in Egypt: molecular and pathological characterization. Tropical animal health and production, 52: 1179-1186.

Abraham, S. S., Asha, T., Julie, B., Prathiush, P., Nandakumar, S., \& Prasad, P. (2015). Pathological and molecular characterization of contagious caprine pleuropneumonia (CCPP) outbreak in Kerala. Indian Journal of Veterinary Pathology, 39: 121-124.

Ahmad, F., Khan, H., Khan, F. A., Carson, B. D., Sadique, U., Ahmad, I., Rehman, H. U. (2020). The first isolation and molecular characterization of Mycoplasma capricolum subsp. capripneumoniae Pakistan strain: A causative agent of contagious caprine pleuropneumonia. Journal of Microbiology, Immunology, and Infection.

Ejaz, H., Hashmi, H., Awan, M. A., Kakar, M. A., Shahwani, M. N., Ameen, S., \& Tariq, M. M. (2015). Molecular study on the prevalence of respiratory mycoplasma species in small ruminants of Kuchlak, District Quetta and Khanozai, District Pishin, Balochistan. Pakistan Journal of Zoology, 47(2).

El-Deeb, W., Almujalli, A. A., Eljalii, I., Elmoslemany, A., \& Fayez, M. (2017). Contagious caprine pleuropneumonia: The first isolation and molecular characterization of Mycoplasma capricolum subsp. capripneumoniae in the Kingdom of Saudi Arabia. Acta tropica, 168, 74-79.

Hussain, R., Auon, M., Khan, A., Khan, M. Z., Mahmood, F., \& Ur-Rehman, S. (2012). Contagious caprine pleuropneumonia in Beetal goats. Tropical animal health and production, 44(3), 477-481. 
Kabir MH and AS Bari, 2015. Isolation and identification of mycoplasma from respiratory system of goat. Microbiol Res Int, 3: 20-26.

Namazi, F., Derakhshandeh, A., Hezaveh, S. S., \& Eraghi, V. (2020). Detection of Mycoplasma capricolum subsp. capripneumoniae and Mannheimia haemolytica as causative agents of pleuropneumonia in goats. Infectio, 24: 208-211.

OIE, 2008. Contagious caprine pleuropneumonia. In: Terrestrial Manual, Office International des Epizooties 1001-1003.

Parray, O. R., Yatoo, M. I., Bhat, R. A., Malik, H. U., Bashir, S. T., \& Magray, S. N. (2019). Seroepidemiology and risk factor analysis of contagious caprine pleuropneumonia in Himalayan Pashmina Goats. Small Ruminant Research, 171, 23 36.

Peyraud, A., Poumarat, F., Tardy, F., Manso-Silván, L., Hamroev, K., Tilloev, T., \& Thiaucourt, F. (2014). An international collaborative study to determine the prevalence of contagious caprine pleuropneumonia by monoclonal antibody-based cELISA. BMC veterinary research, 10(1), 1-9.

Rahman, H. U., Saddique, U., Shakoor, A., Shah, M. K., Shah, S. S. A., Khan, F. A., ur Rahman, S. (2018). The predominant incidence of Mycoplasma mycoides subsp. capri in suspected cases of contagious caprine pleuropneumonia in sheep and goats of northern Pakistan. Pakistan Journal of Zoology, 50.

Shah, M.K., Saddique, U., Ahmad, S., Iqbal, A., Ali, A., Shahzad, W., S. S. Ali Shah, (2017). Molecular Characterization of Local Isolates of Mycoplasma capricolum Sub Specie Capripneumoniae in Goats (Capra hircus) of Khyber Pakhtunkhwa, Pakistan. Pakistan Veterinary Journal, 37(1).

Shahzad, W., R. Munir, M.S. Khan, et al., 2012. Characterization, molecular diagnosis and prevalence of Caprine mycoplasmosis in different areas of Pakistan. Pak J Zool, 44: 559-568.

Shahzad, W., Yaqoob, T., Mukhtar, N., Munir, R., Ahmad, R., Khan, M. S., \& Hussain, A. (2016). sero-prevalence of mycoplasma capricolum subsp. capripneumoniae in goats through cELISA in different districts of punjab, Pakistan. The Journal of Animal \& Plant Sciences, 26(4), 931-937.

Teshome, D., Sori, T., Sacchini, F., \& Wieland, B. (2019). Epidemiological investigations of contagious caprine pleuropneumonia in selected districts of Borana zone, Southern Oromia, Ethiopia. Tropical animal health and production, 51(3), 703-711.

Thakur, S., Sharma, M., Verma, S., \& Kanwar, P. (2019). Confirmation of contagious caprine pleuropneumonia outbreak in Gaddi goats of Himachal Pradesh, India. Indian Journal of Comparative Microbiology, Immunology and Infectious Diseases, 40(1), 21-30.

Woubit, S., Lorenzon, S., Peyraud, A., Manso-Silvan, L., \& Thiaucourt, F. (2004). A specific PCR for the identification of Mycoplasma capricolum subsp. capripneumoniae, the causative agent of contagious caprine pleuropneumonia (CCPP). Veterinary microbiology, 104(1-2), 125-132. 